\title{
First Report of Pyoderma Gangrenosum after Surgery of Breast Cancer-Related Lymphedema with Transfer of Vascularized Free Lymph Nodes of the Groin and Simultaneous DIEP Flap
}

\author{
Angela A. García-Ruano Esther Deleyto Jose Maria Lasso
}

Hospital Gregorio Marañón, Madrid, Spain

\section{Established Facts}

- Although infrequently, Pyoderma gangrenosum (PG) can appear after any kind of trauma or injury to the skin (pathergy process), as occurs during a surgical procedure.

- PG should be considered as a possible cause of a postsurgical ulcerative wound that does not respond to antibiotics and worsens with surgical debridement.

- Diagnosis of PG is often delayed because of lack of clinical suspicion. It is only confirmed by an intense response to immunosuppressive treatment.

\section{Novel Insights}

- MPG can occur as a complication of the microsurgical treatment of breast cancer-related lymphedema.

- Surgical repair of the skin lesions of PG is secure if performed under steroid coverage.

\section{Keywords}

DIEP · Lymph node · Pyoderma gangrenosum .

Autoimmune disease

\section{Summary}

Background: Pyoderma gangrenosum (PG) is a rare cutaneous ulcerative disease that does not respond to antibiotics and worsens with surgical manipulation. This is the first report in the literature revealing that this disease complicates total microsurgical breast reconstruction associated with the transfer of free lymph nodes. Case Report: We report the case of a female patient who underwent left-breast microsurgical reconstruction with a deep inferior epigastric perforator (DIEP) flap and simultaneous transfer of vascularized free lymph nodes for the surgical treatment of second- ary left upper-limb lymphedema, and who developed a severe PG of both the skin of the flap and the donor zone on postoperative day 7. After exclusion of other etiologies, treatment with high-dose corticosteroids and topical zinc sulfate was initiated. The flap was salvaged and breast cancer-related lymphedema (BCRL) surgery was definitively effective despite the PG. Conclusions: PG should be considered in the differential diagnosis of progressive cutaneous ulcers following surgical interventions, once infectious and ischemic etiologies have been excluded. Early initiation of immunosuppressive treatment can lead to preservation of the initial flap and function of the transferred lymph nodes in the case of microsurgical breast reconstruction with associated lymph node transfer.

(c) 2016 S. Karger GmbH, Freiburg

\section{KARGER}

Fax +497614520714

\section{(c) 2016 S. Karger GmbH, Freiburg}

$1661-3791 / 16 / 0111-0057 \$ 39.50 / 0$ 
Fig. 1. Preoperative images.

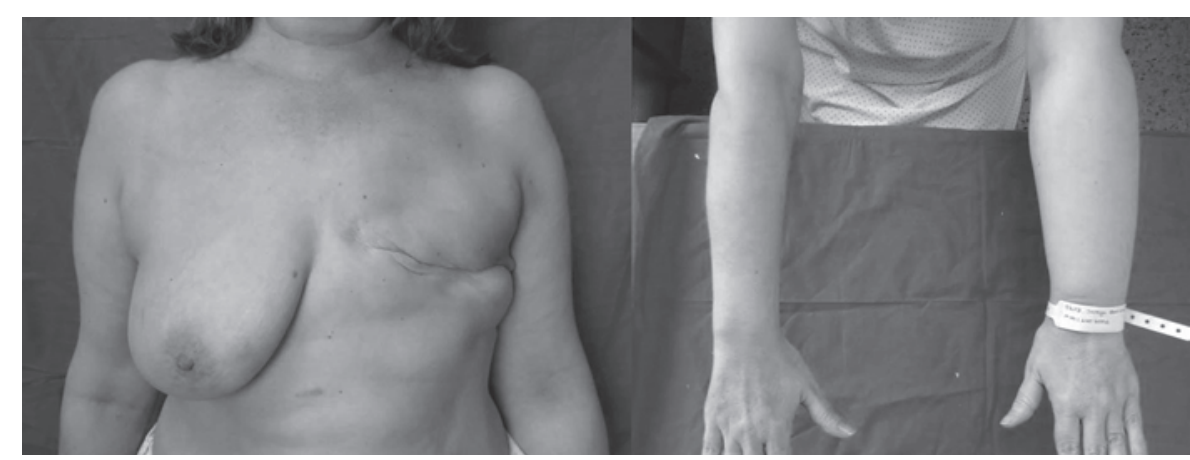

\section{Introduction}

Pyoderma gangrenosum (PG) is a rare cutaneous ulcerative disease of autoimmune etiology. Its clinical presentation is characterized by a progressive ulceration of the skin that does not respond to antibiotics and worsens with surgical manipulation [1]. Histological findings show neutrophilic infiltrates [2]. As both the clinical and histological features are non-specific, this disease is rarely suspected. Diagnosis is confirmed by an intense response to immunosuppressive treatment.

We present the first case report in the literature of PG associated with surgical treatment of breast cancer-related lymphedema (BCRL), and we highlight the importance of suspicion of PG when presented with a postoperative wound that does not respond to standard initial care.

\section{Case Report}

We report the case of a 45-year-old patient with invasive breast cancer treated with modified radical mastectomy and axillary lymph node dissection in February 2010. Complementary treatment with standard chemotherapy, radiotherapy, and hormonotherapy was performed. 7 months after surgery, she complained of progressive left upper-limb lymphedema in relation to a superficial wound on the hand. Non-operative measures were used for management of the lymphedema, including pressotherapy and drainages, with no improvement.

After 21 months, on preoperative evaluation, the physical exam showed irregular mastectomy flaps with a fibrotic scar and grade II left upper-limb BCRL (fig. 1a, b). Lymphoscintigraphy showed no lymphatic stations in the left arm and angiotomography revealed suitable abdominal perforators. Total breast microsurgical reconstruction with a deep inferior epigastric perforator (DIEP) flap with simultaneous lymph node transfer (LNT) to the axillary region was the treatment proposed.

In January 2012, under general anesthesia, the DIEP flap was raised associated with the left superficial inguinal lymph nodes, which were supplied by superficial circumflex vessels. A single anatomical structure with 2 vascular pedicles was obtained and transferred to the thorax. End-to-end microsurgical anastomoses were generated between the internal mammary vessels and the deep inferior epigastric artery and vein, as well as between vessels of the axillary region and the vascular pedicle irrigating the lymph node group. The lymph nodes were placed close to the axillary vein.

There were no complications during the surgical procedure, which was completed in $8 \mathrm{~h}$. On postoperative day 1, the flap showed good viability signs, but a hemorrhagic suffusion appeared at its lateral border. The day after, it turned into a blister, which was attributed to flap congestion in zone IV. On the 3rd postoperative day, the patient presented with fever despite antibiotic therapy (cefazoline), with no associated symptoms. The flap still maintained good

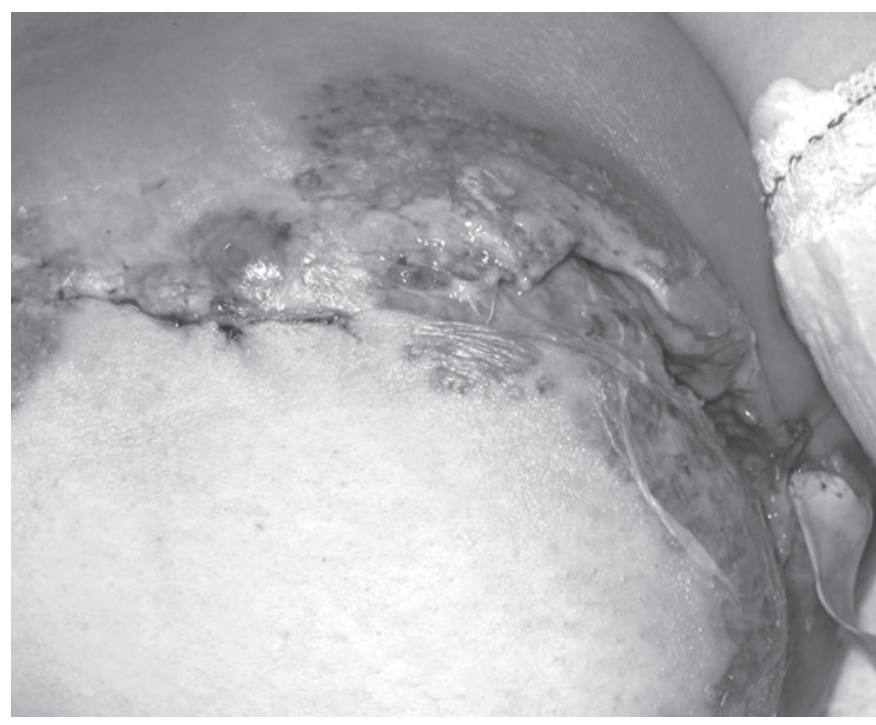

Fig. 2. Severe cutaneous affectation of the flap.

viability signs but the skin lesions were more extensive. Antibiotic coverage was broadened with ciprofloxacin, still without response. On the following days, the fever continued, now with hemodynamic compromise. Skin lesions evolved to pustules with yellowish content and ulcers appearing on the medial side of the flap and at the adjacent native thoracic skin, as well as at the abdominal surgical site. A progressive erythematous halo with cellulitis extended from the affected sites and progressed outwards. Examination of the peripheral blood displayed normal results, but a computed tomography scan showed left pleural effusion.

Suspecting necrotizing fascitis, conservative surgical debridement was performed with preservation of the central portion of the flap and respecting the transplanted lymph nodes. Intraoperative biopsies were taken for microbiological culture, and empiric treatment with meropenem, clindamycin, and vancomycin was prescribed. On the 8 th postoperative day, the patient was admitted into the intensive care unit, with severe hemodynamic compromise, high fever, and progressive flap necrosis, refractory to every treatment (fig. 2).

Cultures and the group A streptococcus rapid test were negative. The chart was reconsidered for other possible diagnoses and the possibility of a PG emerged. Skin biopsies were taken from the affected skin for histological studies. One of the samples was described as compatible with PG, with a diffuse dermal neutrophilic infiltrate. Immediately, steroid treatment with intravenous methylprednisolone $16 \mathrm{mg} / 8 \mathrm{~h}$ was established. On the 12th postoperative day, the fever disappeared as did the erythematous halo, and the hemodynamic status stabilized. Topic treatment with zinc sulfate $1 / 1,000$ was applied to the lesions. From this point, the patient underwent slow but continuous improvement. On postoperative day 25 , under steroid coverage, skin closure of the surgical sites was performed. The patient was discharged from the hospital 1 month after the initial surgery, with a cosmetically acceptable reconstructed breast and transplantation of functioning lymph nodes. Ambulatory oral ster- 


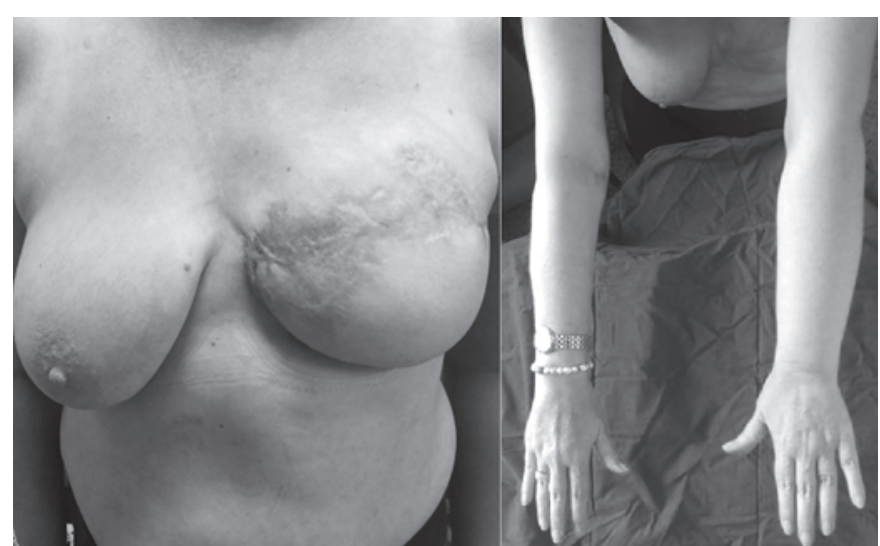

Fig. 3. Postoperative images. Objective reduction of the circumference measures between both arms can be noted, which was $13 \mathrm{~cm}$ at the 11th month postsurgery compared to $21 \mathrm{~cm}$ preoperatively.

oid treatment (prednisone $40 \mathrm{mg} /$ day) was continued for 6 months. Follow-up showed subjective improvement of the lymphedema with less pain and heaviness as well as objective reduction of the circumference measures at different points of the affected arm (fig. 3), even during corticotherapy.

\section{Discussion}

PG is a rare ulcerative disease first described in 1930 by Brunsting et al. [3]. Despite this, its pathogenesis remains unknown, although it is considered an autoimmune disorder. In $50 \%$ of the cases, PG is associated with systemic diseases, especially inflammatory bowel disease [4], but up to $25 \%$ of cases occur as an isolated process. The characteristic lesions are papules evolving to pustules and skin ulcers enlarging centripetally. In many cases, PG presents as painful. It can, however, be painless, particularly in cases with reduced local sensibility, as seen in autologous breast reconstructions. In $30 \%$ of cases, the disease occurs after trauma or injury to the skin, such as occurs in a surgical procedure, due to the process of pathergy. There has only been a limited number of case reports of PG complicating breast surgery [5, 6], most of them referring to reduction mammaplasties and the latest ones in cases of microsurgical breast reconstruction. However, there is no previous report in the literature involving lymphatic microsurgery, as in our case.
LNT is an accepted treatment of secondary lymphedema that consistently reduces heaviness of and pain in the affected limb, as well as its circumference [7]. After BCRL, chimeric flaps involving the DIEP flap and vascularized groin LNT are an effective and complete therapeutic option for the management of this condition [8].

In our experience, infectious complications of a microvascularized flap are uncommon. However, the clinical evolution and the skin affectation observed in our patient suggested the diagnosis of PG. As there are no laboratory parameters for PG, and since the histopathology varies with the stage of the dermatosis, PG remains an elusive diagnosis made by exclusion after other etiologies have been ruled out, as in our case. Response to a high-dose prednisone course confirms the diagnosis. With respect to surgical debridement, some authors advise against it, letting lesions heal by secondary intention with significant scarring [5], while others advocate for surgical revision under steroid coverage [1]. In our case, immunosuppressive treatment with methylprednisolone improved the clinical status of our patient, stabilized the cutaneous lesions and allowed secondary surgical revision to minimize scarring. Also, this management effectively preserved the transferred lymph nodes in our patient, which remained functional with continued improvement of the lymphedema over the following months.

\section{Conclusions}

PG should be considered in the differential diagnosis of progressive cutaneous ulcers that do not respond to standard initial care following a surgical intervention. Once infectious and ischemic etiologies have been excluded, response to immunosuppressive management confirms the suspicion of PG. Early diagnosis of the disease and initiation of treatment with high-dose corticosteroids can lead to preservation of the initial flap and of the function of the transferred lymph nodes in cases of microsurgical breast reconstruction with associated LNT.

\section{Disclosure Statement}

None declared.

\section{References}

1 Rand RP, Brown GL, Bostwick III J: Pyoderma gangrenosum and progressive cutaneous ulceration. Ann Plast Surg 1988;20:280-284.

$\checkmark 2$ Su WP, Schroeter AL, Perry HO, Powell FC: Histopathologic and immunopathologic study of pyoderma gangrenosum. J Cutan Pathol 1986;13:323-330.

3 Brunsting LA, Goekerman WH, O'Leary PA: Pyoderma gangrenosum: clinical and experimental observations in five cases occurring in adults. Arch Dermatol 1930;22:655-680.
4 Von den Driesch P: Pyoderma gangrenosum: a report of 44 cases with follow-up. Br J Dermatol 1997;137: 1000-1005.

5 Gudi VS, Julian C, Bowers PW: Pyoderma gangrenosum complicating bilateral mammoplasty. Br J Plast Surg 2000;53:440-441.

6 Caterson SA, Nayme T, Phung T, et al.: Pyoderma gangrenosum following bilateral deep inferior epigastric perforator flap breast reconstruction. J Reconstr Microsurg 2010;26:475-479.
Becker C, Assouad J, Riquet M, Hidden G: Postmastectomy lymphedema: long-term results following microsurgical lymph node transplantation. Ann Surg 2006; 263:313-315.

8 Dancey A, Nassimizadeh A, Nassimizadeh M, et al.: A chimeric vascularized groin lymph node flap and DIEP flap for the management of lymphoedema secondary to breast cancer. J Plast Reconstr Aesthet Surg 2013;66: 735-737. 